\title{
Characterization of metabolites in different kiwifruit varieties by NMR and fluorescence spectroscopy
}

\begin{abstract}
It is known from our previous studies that kiwifruits, which are used in common human diet, have preventive properties of coronary artery disease. This study describes a combination of 1H NMR spectroscopy, multivariate data analyses and fluorescence measurements in differentiating of some kiwifruit varieties, their quenching and antioxidant properties. A total of 41 metabolites were identified by comparing with literature data Chenomx database and 2D NMR. The binding properties of the extracted polyphenols against HSA showed higher reactivity of studied two cultivars in comparison with the common Hayward. The results showed that the fluorescence of HSA was quenched by Bidan as much as twice than by other fruits. The correlation between the binding properties of polyphenols in the investigated fruits, their relative quantification and suggested metabolic pathway was established. These results can provide possible application of fruit extracts in pharmaceutical industry.
\end{abstract}

Keyword: NMR spectroscopy; Metabolites in kiwifruits; Fluorescence; Interaction of human serum albumin and polyphenols; Antioxidant and binding properties 